\title{
Successful endoscopic treatment for gastric mural abscess due to fish bone migration
}

\author{
Masabumi Kanekoa, Kyosuke Tanakab, Hiroaki Naotaa, Kazuhiko Kobayashia, \\ Matsusaka Central Hospital, Matsusaka; Mie University Hospital, Tsu, Japan
}

A 50-year-old man was admitted to our hospital because of epigastralgia for one week. He had eaten grilled mackerel before symptom onset. Physical examination showed mild tenderness in the epigastric region without peritoneal signs. Laboratory data demonstrated marked inflammation. Abdominal computed tomography revealed thickening of the gastric wall and an intramural foreign body (Fig. 1). Endoscopy showed reddish swollen mucosa in the gastric antrum (Fig. 2A); however, the foreign body was not exposed inside the stomach. We made a diagnosis of gastric wall abscess due to intramural fish bone migration. We performed endoscopic treatment. A mucosal incision was made with a diathermic knife, after which pus erupted abundantly (Fig. 2B). Pus drainage was facilitated by adjacent mucosal compression around the incision. The intramural fish bone was found through the incision and removed by biopsy forceps (Fig. 2C, D). After the endoscopic treatment, symptoms immediately improved. One month later, endoscopy showed a post-treatment scar with normal adjacent mucosa in the antrum.

Gastric mural abscesses are classified into diffuse and localized types, of which the former is more common [1]. It may be caused by biopsy, polypectomy, cancer or accidental ingestion of foreign objects. Endoscopic ultrasound-guided drainage has been considered useful [2]. However, a case of spontaneous alleviation of gastric mural abscess has also been reported previously [3]. This unusual case shows that making a mucosal incision using a diathermic knife can be an option for the treatment of the localized type of gastric mural abscess due to intramural foreign body migration.

Departments of a Gastroenterology, Matsusaka Central Hospital, Matsusaka (Masabumi Kaneko, Hiroaki Naota, Kazuhiko Kobayashi); 'Endoscopy, Mie University Hospital, Tsu (Kyosuke Tanaka), Japan

\section{Conflict of Interest: None}

Correspondence to: Kyosuke Tanaka MD, PhD, Department of Endoscopy, Mie University Graduate School of Medicine, 2-174 Edobashi, Tsu, Mie, 514-8507 Japan, Tel.: +81 59231 5016,

Fax: +81 59231 5200, e-mail: kyosuket@clin.medic.mie-u.ac.jp

Received 03 June 2017; accepted 13 June 2017; published online 14 July 2017

DOI: https://doi.org/10.20524/aog.2017.0177

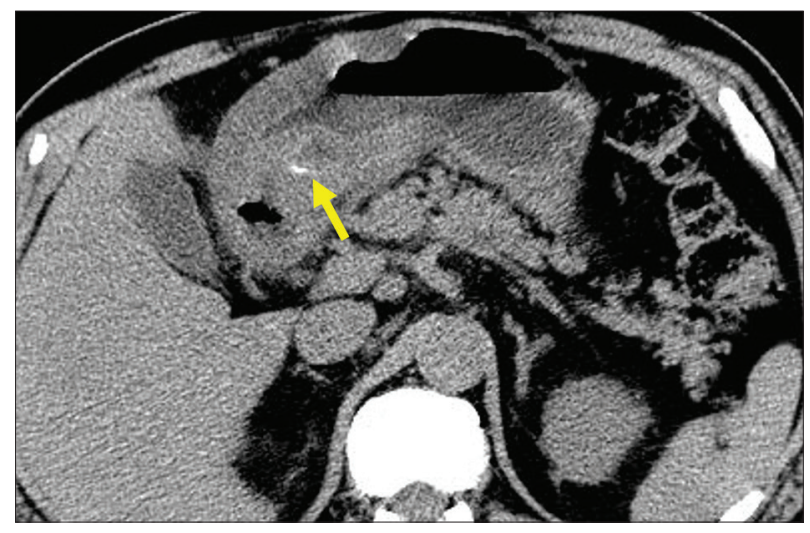

Figure 1 Abdominal computed tomography revealed thickening of the gastric wall and an intramural foreign body (arrow)

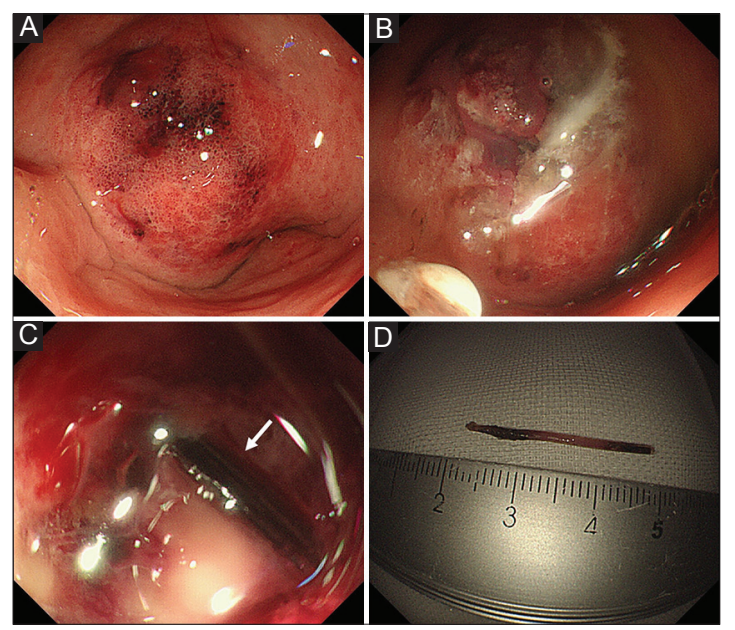

Figure 2 (A) Endoscopy showed reddish swollen mucosa in the minor curvature of the gastric antrum. (B) A mucosal incision was made with a diathermic knife, after which pus erupted abundantly. (C) The intramural fish bone (arrow) was found through the incision. (D) The fish bone removed by biopsy forceps was approximately $25-\mathrm{mm}$ long

\section{References}

1. Chen CH, Yang CC, Yeh YH, Hwang MH. Gastric wall abscess presenting as a submucosal tumor: case report. Gastrointest Endosc 2003;57:959-962.

2. Rana SS, Bhasin DK, Rao C, Gupta R. Endoscopic ultrasoundguided diagnosis and management of an unusual gastric submucosal lesion - gastric wall abscess. Endoscopy 2013;45(Suppl 2 UCTN):E136-E137.

3. Hashimoto R, Chonan A. Spontaneous resolution of intramural gastric abscess without treatment. Ann Gastroenterol 2016;29:227. 